\title{
Vacuum Gauge Performance Verification System
}

\author{
Antons Stekleins, Eriks Gerins \& Artis Kromanis
}
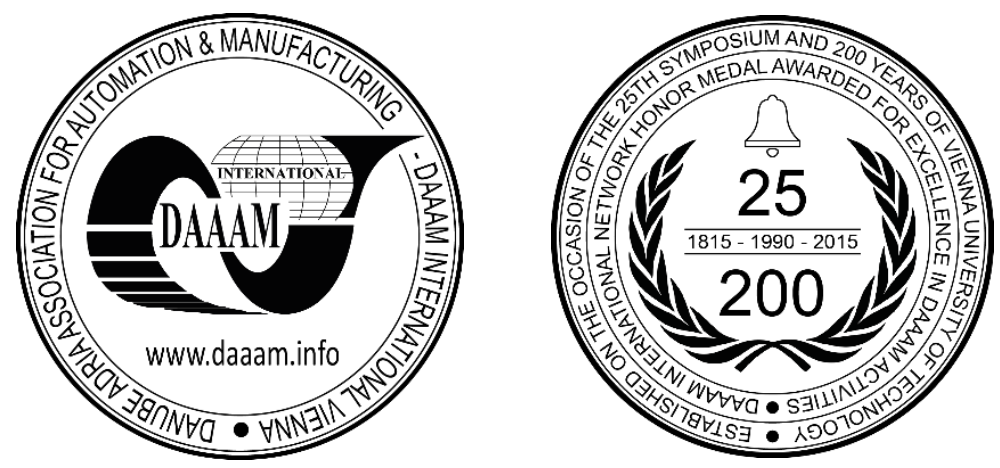

This Publication has to be referred as: Stekleins, A[ntons]; Gerins, E[riks] \& Kromanis, A[rtis] (2016). Vacuum Gauge Performance Verification System, Proceedings of the 27th DAAAM International Symposium, pp.0607-0614, B. Katalinic (Ed.), Published by DAAAM International, ISBN 978-3-902734-08-2, ISSN 1726-9679, Vienna, Austria DOI: $10.2507 / 27$ th.daaam.proceedings.089

\begin{abstract}
The paper analyzes a pressure deviation issues in a vacuum technological process, which occurs due to vacuum gauge inaccuracies during its use. For this purpose a unique vacuum system was designed and used for research. Developed system allows verification of vacuum gauges in manufacturing conditions using various experimental settings for obtaining necessary measuring data and allowing to perform an evaluation about its accuracy, repeatability and the need of calibration. In the following research several experiments with ionization vacuum gauges were carried out to obtain performance data. Presented results and vacuum system allowed to develop a new methodology of vacuum gauge verification under manufacturing conditions.
\end{abstract}

Keywords: vacuum gauge; vacuum system; verification; pressure measurement

\section{Introduction}

One of the vacuum system main requirements is to achieve vacuum in the system and maintain it permanently unchanged to a certain level during the technological process, especially during a coating process. Typically, there are two reasons for providing a vacuum: firstly, to control the reactivity of the deposition process and, secondly, to reduce the number of gas particles collisions thus enabling higher energy particles to be used as part of the deposition process [2].

Vacuum gauge calibration is commonly used when pressure measurement accuracy starts to deviate and it is impossible to secure technological process stability. Some studies analyze vacuum gauge accuracy and calibration period, but there are no clear approaches for vacuum gauge verification under manufacturing conditions before calibration operation. [1,5]. Common to all measuring devices, a pressure-measuring instrumentation needs periodic calibration, primarily to monitor changes in performance [1]. Reliable calibration of gauges is impossible without establishing a widely recognized, true models of accuracy, to which gauges can be verified, also known as "primary vacuum standards" [5]. Therefore we are at the opinion that calibration period must be determined individually by applying specific methodology which includes multifunctional vacuum gauge verification using our developed vacuum system.

In this paper we analyze vacuum gauge unreliable pressure measurements during technological coating process at Sidrabe Inc. and present developed vacuum system designed for vacuum gauge verification under manufacturing conditions. The system provides fast, reliable and multifunctional vacuum gauge verification. Ionization vacuum gauges were tested by comparison method using custom made vacuum system. 
Main purpose of the paper is to propose a new vacuum verification system which can be used in any vacuum coating company to verify vacuum gauges at any moment, even daily to obtain vacuum gauge pressure deviation data.

\section{Vacuum gauge pressure deviation analyses}

At present, there have been identified two core problems associated with the technological coating processes: firstly, a vacuum gauges pressure measurement deviation and, secondly, frequency and the necessity of the calibration.

During manufacturing calibrated vacuum gauges are being used to ensure all necessary product characteristics and quality. In general, the vacuum system contains minimum of two different types of vacuum gauges, which are used to cover several ranges of vacuum, but nevertheless a comparison between two gauges indicated pressure should be performed. Prior art discusses a fact that vacuum gauge accuracy can be influenced by several factors such as: operating conditions (vibration, contamination, and temperature), connection, mounting and corrosive gases [5, 10, 9]. Main problem we have encountered during a daily coating process at Sidrabe Inc. is that the vacuum gauges indicated pressure deviates after vacuum chamber evacuation. The deviation is illustrated in Fig.1.

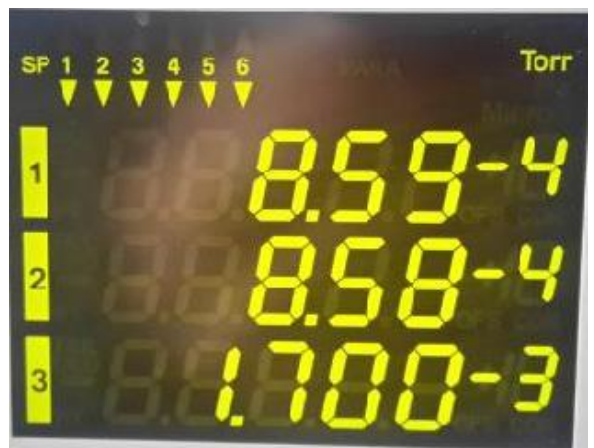

a)

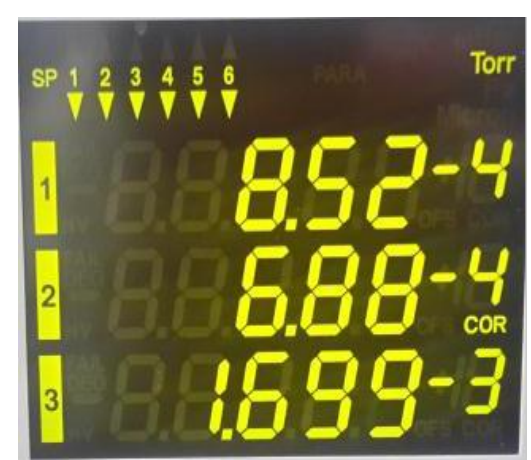

b)

Fig.1. Indicated pressure (a) without argon correction; (b) with argon correction.

Mainly two types of vacuum gauges were used: INFICON® diaphragm vacuum gauge under number three at Fig. 1 (a, b) and ionization gauge HPG 400 under number two at Fig.1 (a,b) [8]. Indicated pressure measurements by two vacuum gauges differ and it makes it even harder to rely on any gauge with $100 \%$ confidence. As it is already known that the diaphragm vacuum gauge is not affected by gas, therefore it is more reliable than ionization gauge [4, 8]. In this experiment as illustrated in Fig.1 (a) an argon is fed into the chamber in volume of $100 \mathrm{sccm}$ or 14,4\%. [8]. In order to reach a pressure measurement without correction and change it from 8,58 up to $6,88^{-4}$ torr, argon must be reduced to 11,2 $\%$ or $78 \mathrm{sccm}[8]$.

If diaphragm vacuum gauge indicated pressure would be considered as true value due to it's resistance to gas, then difference between a true value and ionization gauge indicated pressure with correction turned on would be $59,5 \%$. Current experiment shows used vacuum gauge indicated pressure deviations influence on argon flow amount needed for the process in order to reach required pressure. One of the main problems is that after some time vacuum gauges accuracy start to deviate and it becomes even harder to determine which vacuum gauge indicated correct measurements. Usage of unreliable and inaccurate vacuum gauge may result in following consequences: poor product quality, loss of material, resources and production time for repeating a process (defects). Even minor deviations may leave a significant impact on product characteristics and therefore it's crucial to have reliable vacuum gauge with high accuracy and repeatability. If vacuum system has one vacuum gauge connected, then a determination of gauge pressure precision becomes even more problematic due to lack of comparison operation when two vacuum gauges are connected.

Another issue is related to vacuum gauge calibration necessity and frequency. Prior art discusses that this issue can be addressed with certainty [1]. For majority of instruments the recalibration interval is approximately a year, but if no historical data are available, which relates to the device when used in a manufacturing environment and in a way that is similar to the way it is normally used, then it is not possible to answer the question [1]. Main problem is that during a daily coating process it is possible to reach a point when product characteristics fall outside the permissible limits due to vacuum gauge accuracy failure. Since vacuum gauge operating time, environment inside a vacuum system and other factors are up to the specific coating process, then for the manufacturer it becomes even harder to specify calibration period. If manufacturer suspects that vacuum gauge is defective and unreliable, then the suspected gauge must be disconnected from the vacuum system and sent to the laboratory where it will be calibrated. Usually the manufacturer cannot afford to waste time and resources for calibration operation due to manufacturing intensity, therefore it's necessary to come up with the methodology of vacuum gauge performance verification under production conditions. Using developed methodology would give a possibility to verify a vacuum gauge measurement accuracy, detect deviations in measurements and perform test operations to ensure its accuracy. Since developed system allows all necessary operations to be performed under manufacturing conditions it will facilitate the decision-making about calibration necessity. 


\section{Custom made vacuum verification system}

The aim of the experiment was to build a small size vacuum system in order to reach high vacuum and perform all manipulations needed for vacuum gauge verification using comparison method, gas flow, correction factor and to obtain the necessary data. Custom made vacuum verification system at Sidrabe Inc. can be seen in Fig.2.

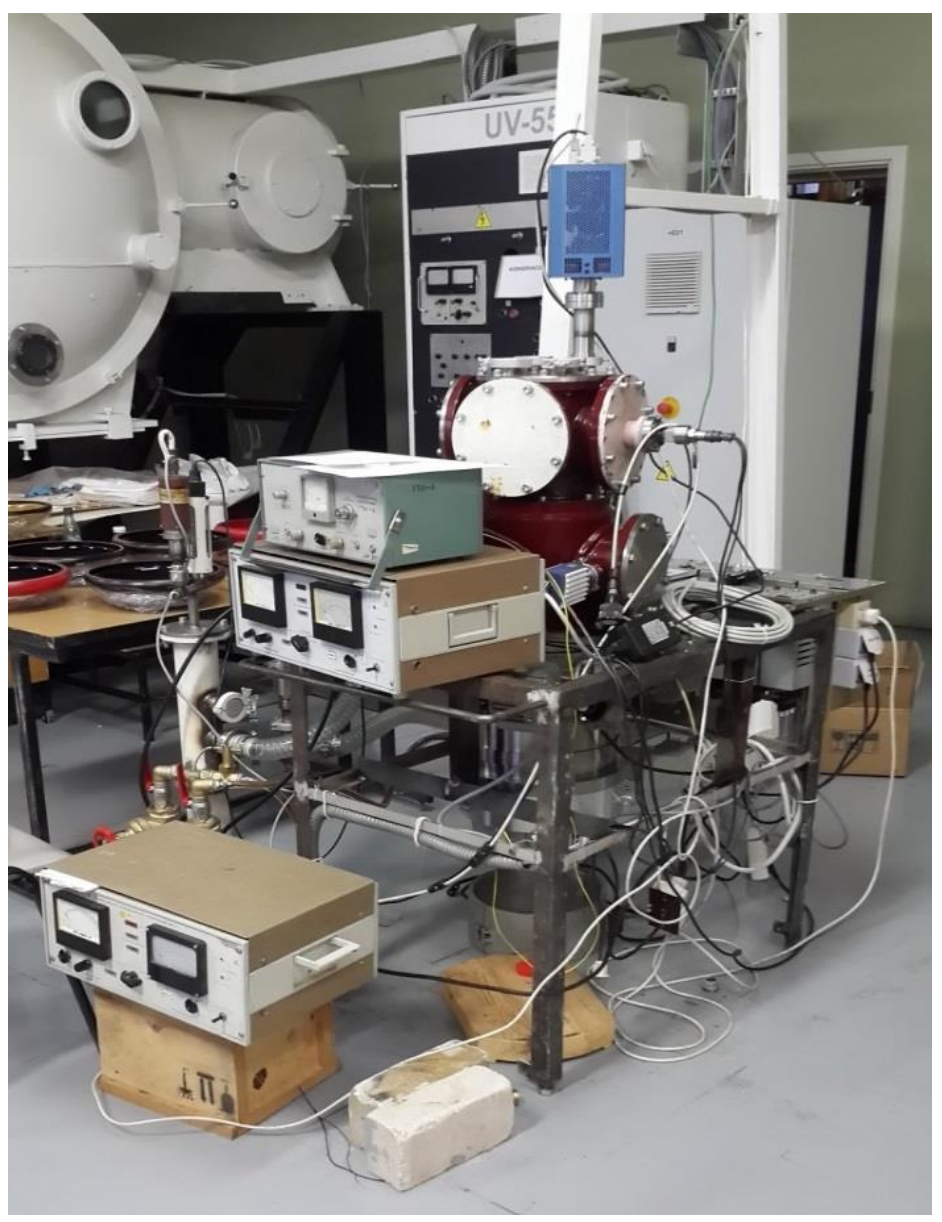

Fig.2. A custom made vacuum verification system.

The vacuum system was design, manufactured and adjusted in two years meeting all selected criteria for fast system evacuation with steady flow of the gas and specific positioning of the vacuum gauges. Vacuum system consists of following components: a chamber, a gate valve, a mechanical pump and a turbo pump, solenoid valves, a manual leak valve, a mass flow controller, flanges with connection ports for vacuum gauges, and a system control unit. Schematic representation of the system is showed in Fig.3.

The system is being controlled manually from control panel and evacuation process is secured by following list of incremental steps. Vacuum system evacuation begins when forevacuum pump receives an input start signal from system control panel and after valves opening provides base pressure of nominally $2 \times 10^{-2}$ Torr. As second step a turbomolecular pump must be enabled, when it accelerates and reaches necessary rotational speed. In the meantime the gate valve should be opened. After the gate is opened pumping provides pressure inside vacuum chamber $2 \times 10^{-6}$ Torr. Gas system consists of two separate lines for argon and oxygen flow with manual valves and mass flow controllers in order to control gas flow during experiments. Gas flow can be operated in range from $1 \mathrm{sccm}$ to $10 \mathrm{sccm}$.

One of the most important parameters of vacuum system is the design of vacuum chamber. Manufactured vacuum system is capable of providing the lowest pressure of $8 \times 10^{-7}$ Torr. Given pressure can be achieved in short period of time comparing to the coating vacuum systems with high volume for manufacturing purpose. Prior art discusses same idea of vacuum calibration system design, where the easiest way to obtain a constant pressure and maintain gas purity in the high vacuum range is to pass a steady flow of the calibration gas in to the vacuum system and out through the high vacuum pump [7]. Given the importance of uniform pressures and the number of variables that can influence this, it is important to test if the reference gauges and test gauges are sensing the same pressure [7]. Design and the size of verification chamber determine how man vacuum gauges can be mounted simultaneously. Small size vacuum chamber provides faster system evacuation, facilitates to maintain unchanged pressure and significantly reduces the required volume of gas needed. 


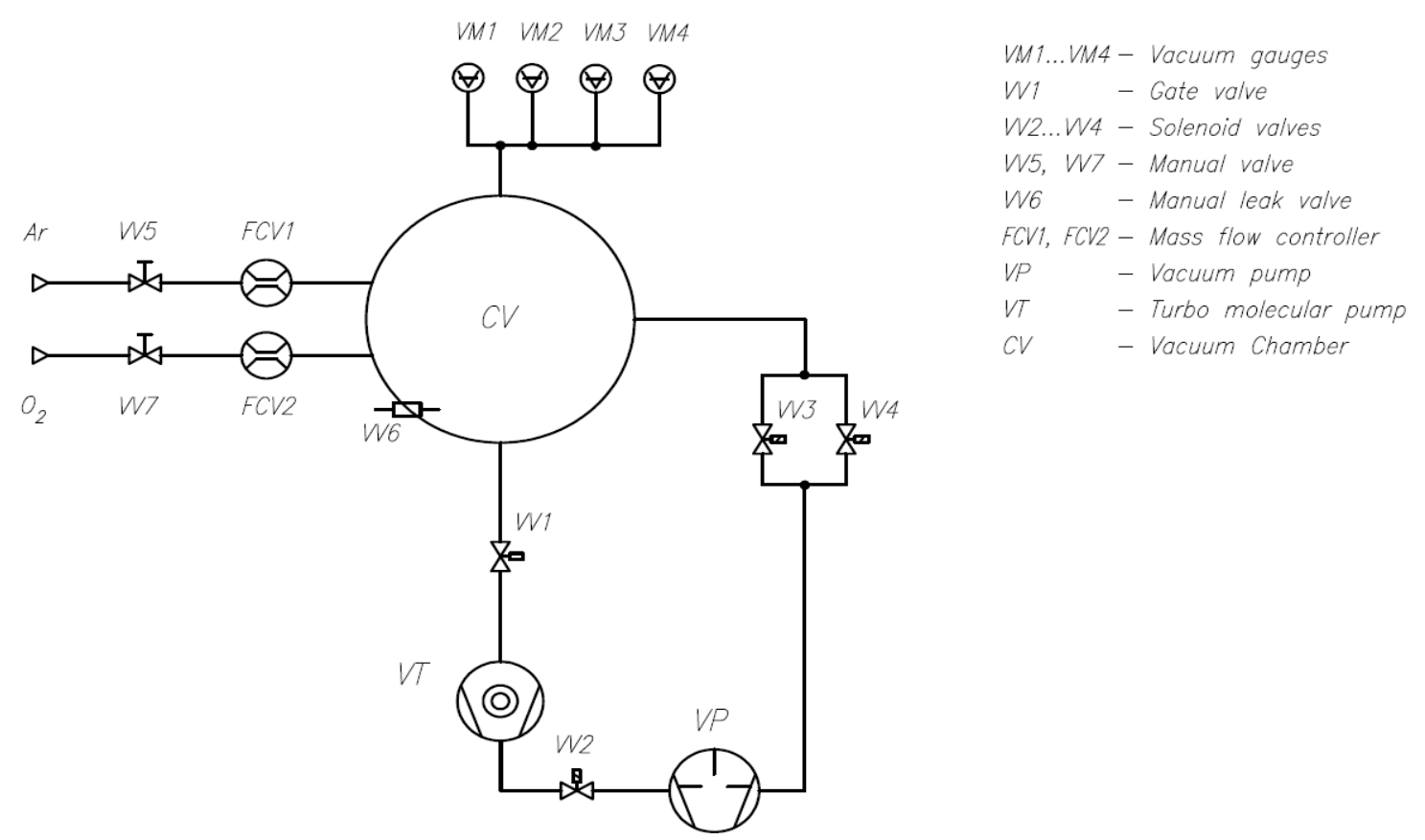

Fig.3. Block scheme of verification vacuum system.

Therefore, several connection ports are necessary to perform vacuum gauges pressure measurement comparison actions, adjustment and calibration if reference standard is available. In order to reach selected criteria the vacuum chamber was designed and manufactured using mechanical machining and blacksmithing work. All the vacuum system components are showed at fig. 4.
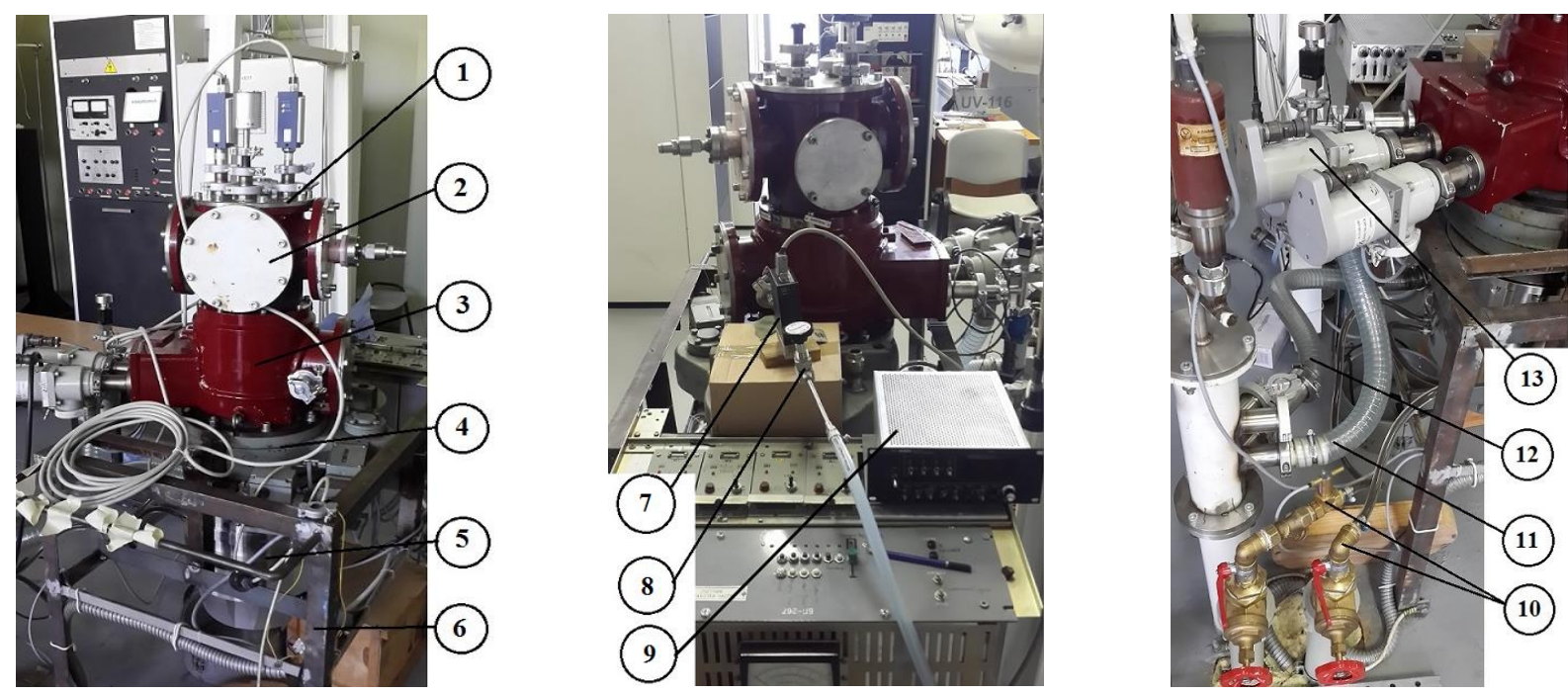

Fig. 4. Vacuum verification system with its components.

As illustarted there are four side covers and one cover at the top of the chamber for vacuum gauges connections. Main idea was to design relatively large connection ports with covers, place them symmetrically to each other. Such design allows to connect up to four vacuum gauges on one cover, thus a distance from pumping orifice and gas inlet port is equal, which provides reliable vacuum gauge pressure measurements. This way both vacuum gauges will sense identical pressure in vacuum chamber. For the experiments there were produced several covers, without connections ports and with different connection ports location. Unique design of vacuum chamber lets switch vacuum gauges positions, connect several gauges simultaneously, change position of the gas inlet system. All the manipulations made with listed elements can provide useful information about vacuum gauge performance and pressure measurement accuracy. After preliminary analysis of collected data, it is possible to find relationships between pressure measurements readings, their positioning and gas flow impact.

The system uses turbomolecular pump VARIAN Turbo-V 3K-T (5) connected to a vacuum chamber (1) though a gate valve (4) and having a pumping speed up to $1900 \mathrm{l} / \mathrm{s}$ for argon without inlet screen. This pump consists of high frequency 
motor driving a turbine fitted with 10 bladed stages with nominal rotational speed up to $31800 \mathrm{rmp}$, thus a water-cooling system (10) is essential. Pumping line consists of turbomolecular pump (5) connected to a pipe (12) through solenoid valve and forevacuum pump connected to vacuum chamber through a pipe (11) and through a solenoid valve (13) to vacuum chamber as can be seen at Fig. 4.

Gas system consists of several connected tubes, a manual valve (8), a mass flow controller (7) and a four channel mass flow controller power supply readout (9). Using power supply readout (9), a gas flow ratio can be changed from 1 up to $10 \mathrm{sccm}$. Manual valve is a backup tool for sealing the gas inlet line.

Mainly two types of INFICON vacuum gauges were used in vacuum coating system and for the experiments: a diaphragm vacuum gauge CDG 025D and ionization gauges HPG 400 and BPG 402. Diaphragm vacuum gauge is used as most reliable and stable pressure measuring instrument due to its gas independence. It combines low relative measurement uncertainty with large dynamic range and high stability [6]. Accuracy of gauge also plays significant role and therefore reading uncertainty must be the lowest, thus the diaphragm gauge could meet selected criteria with measurement uncertainty of $0,50 \%$ from reading.

High vacuum must be reached in order to implement vacuum gauge performance verification in broad vacuum range including its upper and lower pressure range limits where relative measurement uncertainty reaches the highest value. Other important factor is that high pressure provides an opportunity for vacuum gauge adjustment. Sometimes the word "calibration" is misused to describe the process of altering the performance of an instrument to ensure that the values it indicates are correct within specified limits [1]. This is adjustment, but not calibration [1]. Formally, calibration is said to be "a set of operations the establish, under specified conditions, the relationship between the values of quantities indicated by a measuring instrument and the corresponding values realized by standards" [1]. Measurement standards can be divided in two different types: primary and secondary [3]. A primary standard is one "designed or widely acknowledged as having the highest metrological qualities and whose value is accepted without reference to other standards of the same quality" [1]. Secondary or transfer standards are calibrated against a primary standard of the same unit and depend on a predictable stability to maintain their accuracy [3]. Vacuum gauge calibration in manufacturing conditions cannot be made due to several reasons including the reference standard unavailability; therefore, a vacuum gauge adjustment can be performed. Vacuum gauge operating manual describes that it's recommended to perform a zero adjustment, when gauge is operated for the first time and also due to long time operation or contamination, a zero drift could occur and zero adjustment may become necessary [11]. For adjusting the zero a gauge must be operated under the same constant ambient conditions and in the same mounting orientation as normally [11].

Developed vacuum system was created as a necessary equipment for the vacuum gauges verification under manufacturing conditions in order to test their performance using comparison method, generate adjustment, obtain and save all the data from experiments, make analysis of relationships between several factors and vacuum gauge pressure measurements accuracy.

\section{Experiments}

In research of vacuum gauge performance, several experiments were designed and conducted using developed verification system. As the first step a vacuum gauge pressure measurements values can be checked by comparison method. For the experiment it was necessary to select minimum of two identical vacuum gauges and third one as a reference gauge. In this particular experiment two INFICON ionization gauges HPG 400 were selected as verifiable gauges and third gauges BPG 402 was selected as a reference.

\begin{tabular}{|c|c|c|c|}
\hline No. & HPG 400 (SN 566) & BPG 402 & HPG 400 (SN 549) \\
\hline 1 & $2,50 \mathrm{E}-03$ (Torr) & $1,90 \mathrm{E}-03$ (Torr) & $8,00 \mathrm{E}-04$ (Torr) \\
\hline 2 & $1,00 \mathrm{E}-03$ (Torr) & $8,00 \mathrm{E}-04$ (Torr) & $3,20 \mathrm{E}-04$ (Torr) \\
\hline 3 & $2,10 \mathrm{E}-04$ (Torr) & $1,70 \mathrm{E}-04$ (Torr) & $6,50 \mathrm{E}-05$ (Torr) \\
\hline 4 & $5,90 \mathrm{E}-05$ (Torr) & $5,30 \mathrm{E}-05$ (Torr) & $2,20 \mathrm{E}-05$ (Torr) \\
\hline 5 & $3,20 \mathrm{E}-05$ (Torr) & $3,00 \mathrm{E}-05$ (Torr) & $1,40 \mathrm{E}-05$ (Torr) \\
\hline 6 & $1,30 \mathrm{E}-05$ (Torr) & $1,40 \mathrm{E}-05$ (Torr) & $7,50 \mathrm{E}-06$ (Torr) \\
\hline 7 & $8,20 \mathrm{E}-06$ (Torr) & $1,00 \mathrm{E}-05$ (Torr) & $5,90 \mathrm{E}-06$ (Torr) \\
\hline 8 & $4,60 \mathrm{E}-06$ (Torr) & $7,10 \mathrm{E}-06$ (Torr) & $4,50 \mathrm{E}-06$ (Torr) \\
\hline 9 & $1,80 \mathrm{E}-06$ (Torr) & $4,00 \mathrm{E}-06$ (Torr) & $2,70 \mathrm{E}-06$ (Torr) \\
\hline
\end{tabular}

Table 1. HPG and BPG vacuum gauges measured pressures values. 


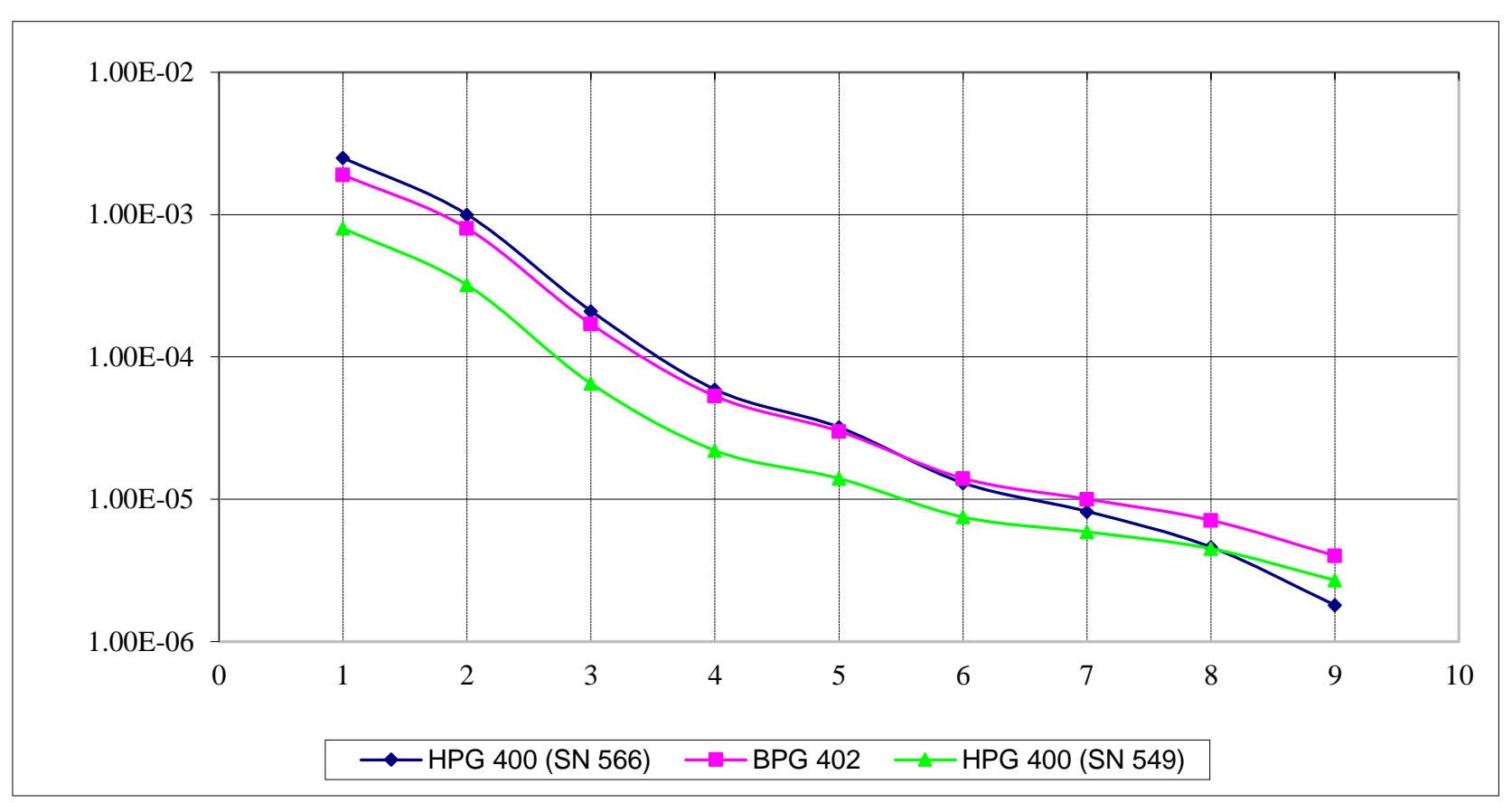

Fig.5. HPG 400 and BPG 402 vacuum gauge performance comparison.

In INFICON HPG 400 ionization vacuum gauge data sheets stated that the accuracy is $+/-15 \%$ of reading in range from 10E-5 to 1 mbar [12]. Same accuracy provided by the BPG 402 gauges in the range from 10E-8 to 10E-2 mbar [13]. After preliminary analysis of the results it was possible to conclude that ionization gauge HPG 400 (SN 549) lacks accuracy due to significant difference in pressure measurements comparing to reference gauge. As an example, the first measuring point was compared for HPG 400 (SN549) and BPG 402 gauges. Difference between indicated pressures was $58 \%$ if we accept that reference gauge pressure is a true value. Prior art discusses that in order to be able to allocate the largest possible measuring ranges to the individual types of vacuum gauge, one has to accept the fact that the measurement uncertainty rises very rapidly, in some cases up to the greater extent, particularly at the upper and lower limit [5]. First three measuring points do not exceed upper limit for HPG 400 ionization gauges, thus accuracy at specified pressure range complies lowest possible uncertainty. BPG type vacuum gauge accuracy at all measuring points remains the highest due to its relative measurement uncertainty in the middle of range limits.

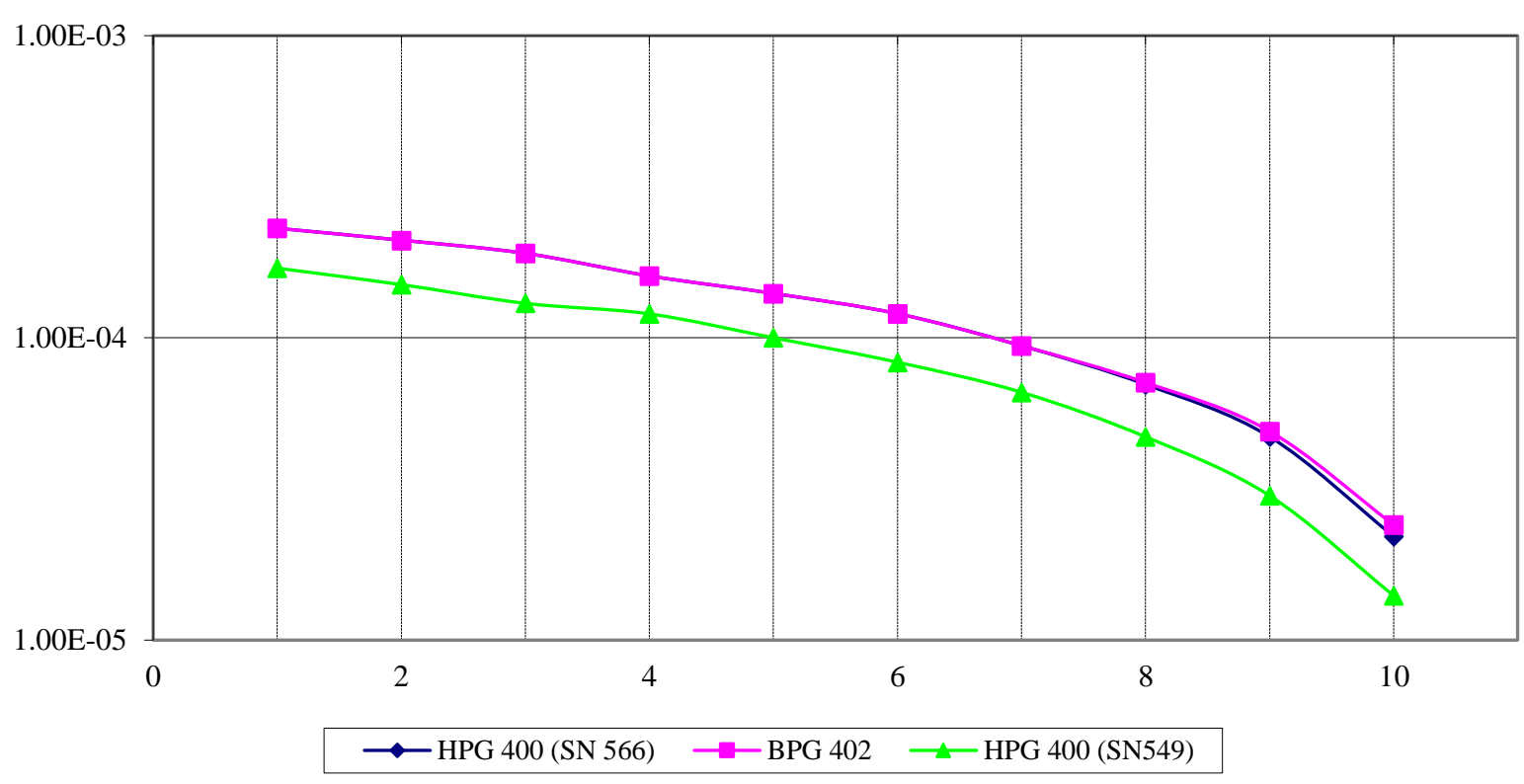

Fig.6. HPG 400 and BPG 402 vacuum gauge performance comparison with argon gas. 


\begin{tabular}{|c|c|c|c|c|}
\hline No. & Argon flow (sccm) & HPG 400 (SN 566) & BPG 402 & HPG 400 (SN549) \\
\hline 1 & $10 \mathrm{sccm}$ & $2,30 \mathrm{E}-04$ & $2,30 \mathrm{E}-04$ & $1,70 \mathrm{E}-04$ \\
\hline 2 & $9 \mathrm{sccm}$ & $2,10 \mathrm{E}-04$ & $2,10 \mathrm{E}-04$ & $1,50 \mathrm{E}-04$ \\
\hline 3 & $8 \mathrm{sccm}$ & $1,90 \mathrm{E}-04$ & $1,90 \mathrm{E}-04$ & $1,30 \mathrm{E}-04$ \\
\hline 4 & $7 \mathrm{sccm}$ & $1,60 \mathrm{E}-04$ & $1,60 \mathrm{E}-04$ & $1,20 \mathrm{E}-04$ \\
\hline 5 & $6 \mathrm{sccm}$ & $1,40 \mathrm{E}-04$ & $1,40 \mathrm{E}-04$ & $1,00 \mathrm{E}-04$ \\
\hline 6 & $5 \mathrm{sccm}$ & $1,20 \mathrm{E}-04$ & $1,20 \mathrm{E}-04$ & $8,30 \mathrm{E}-05$ \\
\hline 7 & $4 \mathrm{sccm}$ & $9,40 \mathrm{E}-05$ & $9,40 \mathrm{E}-05$ & $6,60 \mathrm{E}-05$ \\
\hline 8 & $3 \mathrm{sccm}$ & $7,00 \mathrm{E}-05$ & $7,10 \mathrm{E}-05$ & $4,70 \mathrm{E}-05$ \\
\hline 9 & $2 \mathrm{sccm}$ & $4,70 \mathrm{E}-05$ & $4,90 \mathrm{E}-05$ & $3,00 \mathrm{E}-05$ \\
\hline 10 & $1 \mathrm{sccm}$ & $2,20 \mathrm{E}-05$ & $2,40 \mathrm{E}-05$ & $1,40 \mathrm{E}-05$ \\
\hline
\end{tabular}

Table 2. HPG and BPG vacuum gauges indicated pressures with argon correction.

In the second experiment a comparison of ionization gauges with turned on argon correction was performed. During all experiments an argon flow was controlled and gradually decreased. This step is necessary to obtain a data of vacuum gauges performance with argon correction factor. Another idea for further research is to verify, how vacuum gauge corresponds to gas flow reduction. Ionization gauges HPG 400 (SN566) and BPG 402 graphic lines are identical and indicated pressure values from Table 2 are identical up to eight measurement point, which is upper range limit of HPG 400 (SN 566) where relative measurement uncertainty rises.

Character of pressure measurements changes should be identical in both experiments. From results it was possible to conclude the fact that vacuum gauge performances are identical with and without gas correction factor, but accuracy of ionization vacuum gauge HPG 400 (SN 549) exceeds the permissible limits. As an example the sixth measuring point can be compared for HPG 400 (SN549) and BPG 402 gauges. Difference between reference and tested gauge at sixth point indicated $31 \%$ of deviation. HPG 400 gauges consists of two subgauges, where Pirani gauge filament is exposed to vacuum, thus during opening of a chamber and during technological process, the particles are deposited on the filament. Contamination of vacuum gauge leads to lack of accuracy and performance characteristics.

\section{Conclusion}

In this paper we analysed vacuum gauge pressure deviation problem. The vacuum system offered in this paper allows performing vacuum gauge verification under manufacturing conditions, adjustment of vacuum in the system, as well as allowing to decide about necessity of calibration period, obtaining and retaining the data. Custom made system has specific design, where vacuum gauges can be tested using various amount of ports and locations, position of gas inlets can be switched, thus small size chamber provides fast system evacuation reaching maximum pressure of 8x10-7 Torr.

Ionization vacuum gauges were tested by comparison method using custom made vacuum system. After preliminary analysis of experimental data it was concluded that ionization vacuum gauge HPG 400 (SN549) lacks accuracy, deviations in measurements are significant compared to reference gauge BPG 402.

This vacuum system can be practically used in any vacuum coating manufacturing company to verify its vacuum gauges, to adjust them, to obtain and retain the data and also make a decision of calibration necessity. This system has unique design which allows further development of various approaches and methods for vacuum gauge verification.

Further research should focus on various approaches and experimental methods which could be implemented for vacuum gauge verification using custom made vacuum system. In result of which a methodology of vacuum gauge verification under manufacturing conditions could be created.

\section{References}

[1] http://www.npl.co.uk, (1998). National Physical Laboratory. Guide to the Measurement of Pressure and Vacuum, Accessed on: 25.09.2016 
[2] Charles A. Bishop. (2007). Vacuum deposition onto Webs, films, and foils, William Andrew Publishing, ISBN-13: 978-0-8155-1535-7 (978-0-8155), 13 Eaton Avenue, Norwich, NY 13815

[3] http://www.glb.nist.gov, 1992. National Institute of Standards and Techonology. Pressure and Vacuum Measurements in Physical Methods of Chemistry, Accessed on 25.09.2016

[4] https://www.leybold.com, 2016. Leybold. Fundamentals of Vacuum Technology , Accessed on 25.09.2016

[5] Akram, H. M., Fasih, A. (2012). Selection criterion of gauges for vacuum measurements of systems with diverse ranges, Physics Procedia, Vol 32., pp. 503 - 512, ISSN: 1875-3892

[6] Barthélémy Daudéa, Hadj Elandaloussia, b, Christof Janssena, b. (2014). On the gas dependence of thermal transpiration and a critical appraisal of correction methods for capacitive diaphragm gauges, Vacuum, Vol. 104, pp. 77 - 87, ISSN: 0042-207X

[7] Ilknur Kocas, Gokce Sevim Sariyerli. 2012. The characterization of new VGMS (vacuum gauge metrology system) by the traceability of multi stage static expansion system, Measurement, Vol. 45, pp., 2430-2433, ISSN: 0263 2241

[8] Stekleins A., Gerins E., Kromanis A. (2016). Analysis of selection criteria for vacuum gauges and its accuracy, unpublished

[9] http://www.lesker.com, 2016. Kurt J.Lesker, 9th edition Global Vacuum Product Guide, Accesses on: 25.09 .2016

[10] http://www.noshok.com 2016, Measurement solutions, Process Conditions That Affect Pressure Guage Accuracy ${ }^{\wedge}$ Performance, Accessed on: 25.092016

[11] http://inficon.com., 2016, Vacuum instruments, CDG 025D Data Sheet, Accessed on: 26.09.2015

[12] http://inficon.com, 2016, Vacuum instruments, HPG 400 Data Sheet, Accessed on: 26.09.2015

[13] http://inficon.com, 2016, Vacuum instruments, BPG 402 Data Sheet, Accessed on: 26.09.2015 